\title{
SITEM for the Conformable Space-Time Fractional (2+1)-Dimensional Breaking Soliton, Third-Order KdV and Burger's Equations
}

\author{
Handan Çerdik Yaslan
}

\begin{abstract}
In the present paper, new analytical solutions for the conformable space-time fractional (2+1)-dimensional breaking soliton, third-order KdV and Burger's equations are obtained by using the simplified $\tan \left(\frac{\phi(\xi)}{2}\right)$ expansion method (SITEM). Here, fractional derivatives are described in conformable sense. The obtained traveling wave solutions are expressed by the trigonometric, hyperbolic, exponential and rational functions. Simulation of the obtained solutions are given at the end of the paper.

Keywords: Space-time fractional (2+1)-dimensional breaking soliton equations; Space-time fractional third-order KdV equation; Space-time fractional Burger's equation; Simplified $\tan \left(\frac{\phi(\xi)}{2}\right)$-expansion method (SITEM).

AMS Subject Classification (2020): Primary: 35C07, 35C08, 35R11.
\end{abstract}

\section{Introduction}

Nonlinear fractional partial differential equations have significant applications in various fields of science and engineering such as fluid mechanics, mechanics of materials, biology, plasma physics, finance, chemistry, image processing (see, for example, [1-5]). Traveling wave solutions to nonlinear fractional partial differential equations play an important role in the study of nonlinear physical phenomena. The traveling wave solutions of the nonlinear partial differential equations have been investigated by using various method such as exponential rational function method, $\left(G^{\prime} / G\right)$-expansion method, Exp-function method, extended sinh-Gordon equation expansion method, modified exponential rational function method, Jacobi elliptic equation method (see, for example,[6-10]).

$(2+1)$-dimensional breaking soliton equations describe the $(2+1)$-dimensional interaction of a Riemann wave propagating along the y-axis with a long wave along the x-axis. $\left(G^{\prime} / G\right)$-expansion method, extended tanh-function method, improved Riccati equations method, sine-cosine method, improved extended Fan sub-equation method, generalized $\left(G^{\prime} / G\right)$-expansion method and extended three wave method have been applied to the (2+1)-dimensional breaking soliton equations [11-18]. The space-time fractional $(2+1)$-dimensional breaking soliton equations with modified Riemann-Liouville derivative have been solved by using new fractional Jacobi elliptic equation method, 
new fractional sub-equation method, modified simple equation method, improved fractional sub-equation method, exponential rational function method, new fractional Jacobi elliptic equation method [19-23]. $\left(G^{\prime} / G\right)$-expansion method has been studied for the space fractional $(2+1)$-dimensional breaking soliton equations with modified Riemann-Liouville derivative [24].

The general projective Riccati equation method, Exp-function method, extended hyperbolic function method and collocation method with the modified exponential cubic B-spline have been applied to the third-order KdV equation [25-28]. Time-fractional generalized third-order KdV equation with modified Riemann-Liouville derivative has been solved by using generalized Kudryashov method [29].

Burger's equation plays a major role in the study of nonlinear waves since it is used as a mathematical model in turbulence problems, in the theory of shock waves, and in continuous stochastic processes [30]. HopfCole transformation and a reproducing kernel function method, a semi-analytical iterative method, $\left(G^{\prime} / G, 1 / G\right)$ expansion method have been applied to the Burger's equation [31-33].

In this paper, the conformable space-time fractional (2+1)-dimensional breaking soliton, third-order $\mathrm{KdV}$ and Burger's equations have been solved by using the simplified $\tan \left(\frac{\phi(\xi)}{2}\right)$-expansion method (SITEM). SITEM has been applied to the Kundu-Eckhaus equation only for the parameter $p=0$ in [34]. In our work, SITEM for the nonzero parameter $p$ has been applied to the space-time fractional some evolution equations with conformable fractional derivative. New analytic solutions for these equations have been reported. Note that space-time fractional $(2+1)$-dimensional breaking soliton, third-order KdV and Burger's equations including conformable derivatives have not yet been solved.

\section{Description of the conformable fractional derivative and its properties}

For a function $f:(0, \infty) \rightarrow R$, the conformable fractional derivative of $f$ of order $0<\alpha<1$ is defined as (see, for example, [35])

$$
T_{t}^{\alpha} f(t)=\lim _{\varepsilon \rightarrow 0} \frac{f\left(t+\varepsilon t^{1-\alpha}\right)-f(t)}{\varepsilon} .
$$

Some important properties of the the conformable fractional derivative are as follows:

$$
\begin{aligned}
T_{t}^{\alpha}(a f+b g)(t) & =a T_{t}^{\alpha} f(t)+b T_{t}^{\alpha} g(t), \forall a, b \in R, \\
T_{t}^{\alpha}\left(t^{\mu}\right) & =\mu t^{\mu-\alpha}, \\
T_{t}^{\alpha}(f(g(t)) & =t^{1-\alpha} g^{\prime}(t) f^{\prime}(g(t)) .
\end{aligned}
$$

\section{Analytic solutions to the conformable space-time fractional (2+1)-dimensional breaking soliton equations}

The breaking soliton equations can be used to describe the $(2+1)$-dimensional interaction of a Riemann wave propagating along the $\mathrm{y}$-axis with a long wave propagating along the $\mathrm{x}$-axis. The $u(x, y, t)$ and $v(x, y, t)$ represent the physical field and some potential, respectively. This equation was studied by Bogoyavenskii [36].

Conformable space-time fractional (2+1)-dimensional breaking soliton equations are given in the following form[23]

$$
\begin{aligned}
& T_{t}^{\alpha} u+T_{x}^{\beta} T_{x}^{\beta} T_{y}^{\theta} u+4 u T_{x}^{\beta} v+4 v T_{x}^{\beta} u=0, \\
& T_{y}^{\theta} u=T_{x}^{\beta} v, \quad 0<\alpha \leq 1, \quad 0<\beta \leq 1,0<\theta \leq 1 .
\end{aligned}
$$

Let us consider the following transformation

$$
u(x, y, t)=U(\xi), v(x, y, t)=V(\xi), \xi=k \frac{t^{\alpha}}{\alpha}+m \frac{x^{\beta}}{\beta}+n \frac{y^{\theta}}{\theta},
$$


where $k, m$ and $n$ are constants. Using the third property in Eq.(2.2), we can compute the following derivatives

$$
\begin{aligned}
& T_{t}^{\alpha} u(x, y, t)=T_{t}^{\alpha} U(\xi)=t^{1-\alpha} \frac{d \xi}{d t} \frac{d U(\xi)}{d \xi}=k U^{\prime}(\xi), \\
& T_{x}^{\beta} u(x, y, t)=T_{x}^{\beta} U(\xi)=x^{1-\beta} \frac{d \xi}{d x} \frac{d U(\xi)}{d \xi}=m U^{\prime}(\xi), \\
& T_{y}^{\theta} u(x, y, t)=T_{y}^{\theta} U(\xi)=y^{1-\theta} \frac{d \xi}{d y} \frac{d U(\xi)}{d \xi}=n U^{\prime}(\xi), \\
& T_{x}^{\beta} T_{x}^{\beta} T_{y}^{\theta} u(x, y, t)=n m^{2} k U^{\prime \prime \prime}(\xi), \\
& T_{x}^{\beta} v(x, y, t)=T_{x}^{\beta} V(\xi)=x^{1-\beta} \frac{d \xi}{d x} \frac{d V(\xi)}{d \xi}=m V^{\prime}(\xi) .
\end{aligned}
$$

Substituting Eqs.(3.4) into Eqs.(3.1)-(3.2), we obtain the following differential equations

$$
\begin{aligned}
& k U^{\prime}+m^{2} n U^{\prime \prime \prime}+4 m U V^{\prime}+4 m V U^{\prime}=0, \\
& n U^{\prime}=m V^{\prime} .
\end{aligned}
$$

Integrating of Eqs.(3.5)-(3.6) with zero constant of integration and eliminating $V$, we have

$$
k U+m^{2} n U^{\prime \prime}+4 n U^{2}=0 .
$$

Let us suppose that the solution of Eq.(3.7) can be expressed in the following form

$$
U(\xi)=\sum_{k=0}^{N} A_{k}\left[p+\tan \left(\frac{\phi(\xi)}{2}\right)\right]^{k}+\sum_{k=1}^{N} B_{k}\left[p+\tan \left(\frac{\phi(\xi)}{2}\right)\right]^{-k} .
$$

Here, $\phi(\xi)$ satisfies the following ordinary differential equation

$$
\phi^{\prime}(\xi)=a \sin (\phi(\xi))+b \cos (\phi(\xi))+c,
$$

and $a, b, c, A_{k}(0 \leq k \leq N)$ and $B_{k}(1 \leq k \leq N)$ are constants to be determined. The solution of Eq. (3.9) is given as follows:

For $b=c, a=0$,

$$
\tan \left(\frac{\phi}{2}\right)=b \xi+c_{1}-p
$$

For $b=c, a \neq 0$,

$$
\tan \left(\frac{\phi}{2}\right)=c_{1} \exp (a \xi)-\frac{b}{a}
$$

For $b \neq c, \Delta=a^{2}+b^{2}-c^{2}>0$,

$$
\tan \left(\frac{\phi}{2}\right)=\frac{2}{b-c} \frac{c_{1} r_{1} \exp \left(r_{1} \xi\right)+c_{2} r_{2} \exp \left(r_{2} \xi\right)}{c_{1} \exp \left(r_{1} \xi\right)+c_{2} \exp \left(r_{2} \xi\right)}-p
$$

For $b \neq c, \Delta=a^{2}+b^{2}-c^{2}=0$,

$$
\tan \left(\frac{\phi}{2}\right)=\frac{a}{b-c}+\frac{2}{b-c} \frac{c_{2}}{c_{1}+c_{2} \xi}
$$

For $b \neq c, \Delta=a^{2}+b^{2}-c^{2}<0$,

$$
\tan \left(\frac{\phi}{2}\right)=\frac{a}{b-c}+\frac{\sqrt{-\Delta}}{b-c} \frac{-c_{1} \sin \left(\frac{\sqrt{-\Delta}}{2} \xi\right)+c_{2} \cos \left(\frac{\sqrt{-\Delta}}{2} \xi\right)}{c_{1} \cos \left(\frac{\sqrt{-\Delta}}{2} \xi\right)+c_{2} \sin \left(\frac{\sqrt{-\Delta}}{2} \xi\right)},
$$

where $c_{1}$ and $c_{2}$ are arbitrary constants, $r_{1}=(a+p(b-c)+\sqrt{\Delta}) / 2$ and $r_{2}=(a+p(b-c)-\sqrt{\Delta}) / 2$. 
Substituting Eq.(3.8) into Eq.(3.7) and then by balancing the highest order derivative term and nonlinear term in result equation, the value of $N$ can be determined as 2. Therefore, Eq.(3.8) reduces to

$$
\begin{aligned}
& U(\xi)=A_{0}+A_{1}\left[p+\tan \left(\frac{\phi(\xi)}{2}\right)\right]+A_{2}\left[p+\tan \left(\frac{\phi(\xi)}{2}\right)\right]^{2} \\
& +\quad B_{1}\left[p+\tan \left(\frac{\phi(\xi)}{2}\right)\right]^{-1}+B_{2}\left[p+\tan \left(\frac{\phi(\xi)}{2}\right)\right]^{-2} .
\end{aligned}
$$

Substituting Eq.(3.10) into Eq.(3.7), collecting all the terms with the same power of $\tan \left(\frac{\phi}{2}\right)$, we can obtain a set of algebraic equations for the unknowns $A_{0}, A_{1}, A_{2}, B_{1}, B_{2}, k, m, n$ :

$$
\begin{aligned}
& 8 n A_{2}^{2}+3 n A_{2} b^{2} m^{2}-6 n A_{2} b c m^{2}+3 n A_{2} c^{2} m^{2}=0, \\
& 64 n p A_{2}^{2}+\ldots
\end{aligned}
$$

Solving the algebraic equations in the Mathematica, we obtain the following set of solutions:

Case 1: $A_{0}=-\frac{3}{8}(b-c) m^{2}\left(-b-c+2 a p+b p^{2}-c p^{2}\right), A_{1}=0, A_{2}=0, B_{1}=\frac{3}{4} m^{2}\left(-a b-a c+2 a^{2} p-b^{2} p+c^{2} p+\right.$ $\left.3 a b p^{2}-3 a c p^{2}+b^{2} p^{3}-2 b c p^{3}+c^{2} p^{3}\right), B_{2}=-\frac{3}{8} m^{2}\left(-b-c+2 a p+b p^{2}-c p^{2}\right)^{2}, k=-\Delta m^{2} n:$

For $b=c$ and $a=0$,

$$
U_{1}(\xi)=-\frac{3}{2} m^{2} b^{2}\left[b \xi+c_{1}\right]^{-2}
$$

For $b=c$ and $a \neq 0$,

$$
\begin{aligned}
& U_{2}(\xi)=\frac{3}{4} m^{2}\left(-2 a b+2 a^{2} p\right)\left[p+c_{1} \exp (a \xi)-\frac{b}{a}\right]^{-1}-\frac{3}{8} m^{2}(-2 b+2 a p)^{2} \\
& \cdot\left[p+c_{1} \exp (a \xi)-\frac{b}{a}\right]^{-2} .
\end{aligned}
$$

For $\Delta>0$ and $b \neq c$,

$$
\begin{aligned}
& U_{3}(\xi)=-\frac{3}{8}(b-c) m^{2}\left(-b-c+2 a p+b p^{2}-c p^{2}\right) \\
+ & \frac{3}{4} m^{2}\left(-a b-a c+2 a^{2} p-b^{2} p+c^{2} p+3 a b p^{2}-3 a c p^{2}+b^{2} p^{3}-2 b c p^{3}+c^{2} p^{3}\right) \\
\cdot & {\left[\frac{2}{b-c} \frac{c_{1} r_{1} \exp \left(r_{1} \xi\right)+c_{2} r_{2} \exp \left(r_{2} \xi\right)}{c_{1} \exp \left(r_{1} \xi\right)+c_{2} \exp \left(r_{2} \xi\right)}\right]^{-1} } \\
- & \frac{3}{8} m^{2}\left(-b-c+2 a p+b p^{2}-c p^{2}\right)^{2}\left[\frac{2}{b-c} \frac{c_{1} r_{1} \exp \left(r_{1} \xi\right)+c_{2} r_{2} \exp \left(r_{2} \xi\right)}{c_{1} \exp \left(r_{1} \xi\right)+c_{2} \exp \left(r_{2} \xi\right)}\right]^{-2} .
\end{aligned}
$$

For $\Delta<0$ and $b \neq c$,

$$
\begin{aligned}
& U_{4}(\xi)=-\frac{3}{8}(b-c) m^{2}\left(-b-c+2 a p+b p^{2}-c p^{2}\right) \\
+ & \frac{3}{4} m^{2}\left(-a b-a c+2 a^{2} p-b^{2} p+c^{2} p+3 a b p^{2}-3 a c p^{2}+b^{2} p^{3}-2 b c p^{3}+c^{2} p^{3}\right) \\
\cdot & {\left[p+\frac{a}{b-c}+\frac{\sqrt{-\Delta}}{b-c} \frac{-c_{1} \sin \left(\frac{\sqrt{-\Delta}}{2} \xi\right)+c_{2} \cos \left(\frac{\sqrt{-\Delta}}{2} \xi\right)}{c_{1} \cos \left(\frac{\sqrt{-\Delta}}{2} \xi\right)+c_{2} \sin \left(\frac{\sqrt{-\Delta}}{2} \xi\right)}\right]^{-1} } \\
- & \frac{3}{8} m^{2}\left(-b-c+2 a p+b p^{2}-c p^{2}\right)^{2}\left[p+\frac{a}{b-c}\right. \\
- & \left.\frac{\sqrt{-\Delta}}{b-c} \frac{-c_{1} \sin \left(\frac{\sqrt{-\Delta}}{2} \xi\right)+c_{2} \cos \left(\frac{\sqrt{-\Delta}}{2} \xi\right)}{c_{1} \cos \left(\frac{\sqrt{-\Delta}}{2} \xi\right)+c_{2} \sin \left(\frac{\sqrt{-\Delta}}{2} \xi\right)}\right]^{-2} .
\end{aligned}
$$

Here $\xi=-\Delta m^{2} n \frac{t^{\alpha}}{\alpha}+m \frac{x^{\beta}}{\beta}+n \frac{x^{\theta}}{\theta}$.

Case 2: $A_{0}=-\frac{1}{8} m^{2}\left(2 a^{2}-b^{2}+c^{2}+6 a b p-6 a c p+3 b^{2} p^{2}-6 b c p^{2}+3 c^{2} p^{2}\right), A_{1}=0, A_{2}=0, B_{1}=\frac{3}{4} m^{2}(-a b-a c+$ $\left.2 a^{2} p-b^{2} p+c^{2} p+3 a b p^{2}-3 a c p^{2}+b^{2} p^{3}-2 b c p^{3}+c^{2} p^{3}\right), B_{2}=-\frac{3}{8} m^{2}\left(-b-c+2 a p+b p^{2}-c p^{2}\right)^{2}, k=\Delta m^{2} n:$ 
For $b=c$ and $a=0$,

$$
U_{5}(\xi)=-\frac{3}{2} m^{2} b^{2}\left[b \xi+c_{1}\right]^{-2}
$$

For $b=c$ and $a \neq 0$,

$$
\begin{gathered}
U_{6}(\xi)=-\frac{1}{4} m^{2} a^{2}+\frac{3}{2} m^{2}\left(-a b+a^{2} p\right)\left[p+c_{1} \exp (a \xi)-\frac{b}{a}\right]^{-1} \\
+-\frac{3}{2} m^{2}(-b+a p)^{2}\left[p+c_{1} \exp (a \xi)-\frac{b}{a}\right]^{-2} .
\end{gathered}
$$

For $\Delta>0$ and $b \neq c$,

$$
\begin{aligned}
& U_{7}(\xi)=-\frac{1}{8} m^{2}\left(2 a^{2}-b^{2}+c^{2}+6 a b p-6 a c p+3 b^{2} p^{2}-6 b c p^{2}+3 c^{2} p^{2}\right) \\
+ & \frac{3}{4} m^{2}\left(-a b-a c+2 a^{2} p-b^{2} p+c^{2} p+3 a b p^{2}-3 a c p^{2}+b^{2} p^{3}-2 b c p^{3}+c^{2} p^{3}\right) \\
\cdot & {\left[\frac{2}{b-c} \frac{c_{1} r_{1} \exp \left(r_{1} \xi\right)+c_{2} r_{2} \exp \left(r_{2} \xi\right)}{c_{1} \exp \left(r_{1} \xi\right)+c_{2} \exp \left(r_{2} \xi\right)}\right]^{-1} } \\
- & \frac{3}{8} m^{2}\left(-b-c+2 a p+b p^{2}-c p^{2}\right)^{2}\left[\frac{2}{b-c} \frac{c_{1} r_{1} \exp \left(r_{1} \xi\right)+c_{2} r_{2} \exp \left(r_{2} \xi\right)}{c_{1} \exp \left(r_{1} \xi\right)+c_{2} \exp \left(r_{2} \xi\right)}\right]^{-2} .
\end{aligned}
$$

For $\Delta<0$ and $b \neq c$,

$$
\begin{aligned}
& U_{8}(\xi)=-\frac{1}{8} m^{2}\left(2 a^{2}-b^{2}+c^{2}+6 a b p-6 a c p+3 b^{2} p^{2}-6 b c p^{2}+3 c^{2} p^{2}\right) \\
+ & \frac{3}{4} m^{2}\left(-a b-a c+2 a^{2} p-b^{2} p+c^{2} p+3 a b p^{2}-3 a c p^{2}+b^{2} p^{3}-2 b c p^{3}+c^{2} p^{3}\right) \\
\cdot & {\left[p+\frac{a}{b-c}+\frac{\sqrt{-\Delta}}{b-c} \frac{-c_{1} \sin \left(\frac{\sqrt{-\Delta}}{2} \xi\right)+c_{2} \cos \left(\frac{\sqrt{-\Delta}}{2} \xi\right)}{c_{1} \cos \left(\frac{\sqrt{-\Delta}}{2} \xi\right)+c_{2} \sin \left(\frac{\sqrt{-\Delta}}{2} \xi\right)}\right]^{-1} } \\
- & \frac{3}{8} m^{2}\left(-b-c+2 a p+b p^{2}-c p^{2}\right)^{2}\left[p+\frac{a}{b-c}\right. \\
+ & \left.\frac{\sqrt{-\Delta}}{b-c} \frac{-c_{1} \sin \left(\frac{\sqrt{-\Delta}}{2} \xi\right)+c_{2} \cos \left(\frac{\sqrt{-\Delta}}{2} \xi\right)}{c_{1} \cos \left(\frac{\sqrt{-\Delta}}{2} \xi\right)+c_{2} \sin \left(\frac{\sqrt{-\Delta}}{2} \xi\right)}\right]^{-2} .
\end{aligned}
$$

Here $\xi=\Delta m^{2} n \frac{t^{\alpha}}{\alpha}+m \frac{x^{\beta}}{\beta}+n \frac{x^{\theta}}{\theta}$.

Case 3: $A_{0}=-\frac{3}{8}(b-c) m^{2}\left(-b-c+2 a p+b p^{2}-c p^{2}\right), A_{1}=\frac{3}{4}(b-c) m^{2}(a+b p-c p), A_{2}=-\frac{3}{8}(b-c)^{2} m^{2}, B_{1}=$ $0, B_{2}=0, k=-\Delta m^{2} n$ :

For $\Delta>0$ and $b \neq c$,

$$
\begin{aligned}
& U_{9}(\xi)=-\frac{3}{8}(b-c) m^{2}\left(-b-c+2 a p+b p^{2}-c p^{2}\right) \\
& +\frac{3}{2} m^{2}(a+b p-c p)\left[\frac{c_{1} r_{1} \exp \left(r_{1} \xi\right)+c_{2} r_{2} \exp \left(r_{2} \xi\right)}{c_{1} \exp \left(r_{1} \xi\right)+c_{2} \exp \left(r_{2} \xi\right)}\right] \\
& -\frac{3}{2} m^{2}\left[\frac{c_{1} r_{1} \exp \left(r_{1} \xi\right)+c_{2} r_{2} \exp \left(r_{2} \xi\right)}{c_{1} \exp \left(r_{1} \xi\right)+c_{2} \exp \left(r_{2} \xi\right)}\right]^{2} .
\end{aligned}
$$

For $\Delta<0$ and $b \neq c$,

$$
\begin{aligned}
& U_{10}(\xi)=-\frac{3}{8}(b-c) m^{2}\left(-b-c+2 a p+b p^{2}-c p^{2}\right) \\
+ & \frac{3}{4} m^{2}(a+b p-c p)\left[p(b-c)+a+\sqrt{-\Delta} \frac{-c_{1} \sin \left(\frac{\sqrt{-\Delta}}{2} \xi\right)+c_{2} \cos \left(\frac{\sqrt{-\Delta}}{2} \xi\right)}{c_{1} \cos \left(\frac{\sqrt{-\Delta}}{2} \xi\right)+c_{2} \sin \left(\frac{\sqrt{-\Delta}}{2} \xi\right)}\right] \\
- & \frac{3}{8} m^{2}\left[p(b-c)+a+\sqrt{-\Delta} \frac{-c_{1} \sin \left(\frac{\sqrt{-\Delta}}{2} \xi\right)+c_{2} \cos \left(\frac{\sqrt{-\Delta}}{2} \xi\right)}{c_{1} \cos \left(\frac{\sqrt{-\Delta}}{2} \xi\right)+c_{2} \sin \left(\frac{\sqrt{-\Delta}}{2} \xi\right)}\right]^{2} .
\end{aligned}
$$


Here $\xi=-\Delta m^{2} n \frac{t^{\alpha}}{\alpha}+m \frac{x^{\beta}}{\beta}+n \frac{x^{\theta}}{\theta}$.

Case 4: $A_{0}=-\frac{1}{8} m^{2}\left(2 a^{2}-b^{2}+c^{2}+6 a b p-6 a c p+3 b^{2} p^{2}-6 b c p^{2}+3 c^{2} p^{2}\right), A_{1}=\frac{3}{4}(b-c) m^{2}(a+b p-c p), A_{2}=$ $-\frac{3}{8}(b-c)^{2} m^{2}, B_{1}=0, B_{2}=0, k=\Delta m^{2} n:$

For $b=c$ and $a \neq 0$,

$$
U_{11}(\xi)=-\frac{1}{4} m^{2} a^{2}
$$

For $\Delta>0$ and $b \neq c$,

$$
\begin{aligned}
& U_{12}(\xi)=-\frac{1}{8} m^{2}\left(2 a^{2}-b^{2}+c^{2}+6 a b p-6 a c p+3 b^{2} p^{2}-6 b c p^{2}+3 c^{2} p^{2}\right) \\
+ & \frac{3}{2} m^{2}(a+b p-c p)\left[\frac{c_{1} r_{1} \exp \left(r_{1} \xi\right)+c_{2} r_{2} \exp \left(r_{2} \xi\right)}{c_{1} \exp \left(r_{1} \xi\right)+c_{2} \exp \left(r_{2} \xi\right)}\right] \\
- & \frac{3}{2} m^{2}\left[\frac{c_{1} r_{1} \exp \left(r_{1} \xi\right)+c_{2} r_{2} \exp \left(r_{2} \xi\right)}{c_{1} \exp \left(r_{1} \xi\right)+c_{2} \exp \left(r_{2} \xi\right)}\right]^{2}
\end{aligned}
$$

For $\Delta<0$ and $b \neq c$,

$$
\begin{gathered}
U_{13}(\xi)=-\frac{1}{8} m^{2}\left(2 a^{2}-b^{2}+c^{2}+6 a b p-6 a c p+3 b^{2} p^{2}-6 b c p^{2}+3 c^{2} p^{2}\right) \\
+\frac{3}{4} m^{2}(a+b p-c p)\left[p(b-c)+a+\sqrt{-\Delta} \frac{-c_{1} \sin \left(\frac{\sqrt{-\Delta}}{2} \xi\right)+c_{2} \cos \left(\frac{\sqrt{-\Delta}}{2} \xi\right)}{c_{1} \cos \left(\frac{\sqrt{-\Delta}}{2} \xi\right)+c_{2} \sin \left(\frac{\sqrt{-\Delta}}{2} \xi\right)}\right] \\
-\frac{3}{8} m^{2}\left[p(b-c)+a+\sqrt{-\Delta} \frac{-c_{1} \sin \left(\frac{\sqrt{-\Delta}}{2} \xi\right)+c_{2} \cos \left(\frac{\sqrt{-\Delta}}{2} \xi\right)}{c_{1} \cos \left(\frac{\sqrt{-\Delta}}{2} \xi\right)+c_{2} \sin \left(\frac{\sqrt{-\Delta}}{2} \xi\right)}\right]^{2} .
\end{gathered}
$$

Here $\xi=\Delta m^{2} n \frac{t^{\alpha}}{\alpha}+m \frac{x^{\beta}}{\beta}+n \frac{y^{\theta}}{\theta}$. Using formula $V(\xi)=\frac{n}{m} U(\xi)$ the unknown function $V(\xi)$ can be computed.

The solutions $u_{2}(x, y, t), u_{3}(x, y, t)$ and $u_{4}(x, y, t)$ of the Eqs.(3.1)-(3.2) are simulated as traveling wave solutions for various values of the physical parameters in Fig.1-Fig.6. Figs.1, 2 show kink waves solutions, Figs.3 and 4 show solitary waves solutions, Figs.5, 6 show periodic waves solutions of Eqs.(3.1)-(3.2). Figs.1 and 2 are 3D and 2D plots of the traveling wave solution $u_{2}(x, 1, t)$ and $u_{2}(x, 1,1)$ in Eq.(3.12)for parameters $\alpha=0.75, \beta=1, \theta=0.5$, $m=-0.05, n=0.5, a=1, b=5, c=5, c_{1}=1, c_{2}=2$ and $p=0.1$. Figs.3 and 4 are 3D and 2D plots of the traveling wave solution $u_{3}(x, 1, t)$ and $u_{3}(x, 1,1)$ in Eq.(3.13) for $\alpha=0.75, \beta=1, \theta=0.5, m=0.5$, $n=0.2, a=0.1, \quad b=0.5, \quad c=0.02, \quad c_{1}=1, \quad c_{2}=1$ and $p=2$. Figs.5 and 6 are 3D and 2D plots of the traveling wave solution $u_{4}(x, 1, t)$ and $u_{4}(x, 1,1)$ in Eq.(3.14) for $\alpha=0.5, \beta=1, \theta=0.5, m=0.5, n=0.2$, $a=0.05, b=0.2, c=0.6, c_{1}=1, c_{2}=1$ and $p=1$. Note that the 3D graphs describe the behavior of $u$ in space $x$ and time $t$ at fixed $y=1$, which represents the change of amplitude and shape for each obtained traveling wave solutions. 2D graphs describe the behavior of $u$ in space $x$ at fixed time $t=1$ and fixed $y=1$. All graphics in figures are drawn by the aid of Mathematica 10.

\section{Analytic solutions to the conformable space-time fractional Korteweg-de Vries (KdV) equation}

Conformable space-time fractional $\mathrm{KdV}$ equation is given in the following form[25]

$$
T_{t}^{\alpha} u+T_{x}^{\beta} T_{x}^{\beta} T_{x}^{\beta} u+6 u T_{x}^{\beta} u=0,0<\alpha \leq 1,0<\beta \leq 1 .
$$

Let us consider the following transformation

$$
u(x, t)=U(\xi), \xi=k \frac{t^{\alpha}}{\alpha}+m \frac{x^{\beta}}{\beta},
$$

where $k, m$ are constants. Substituting (4.2) into Eq.(4.1) we obtain the following differential equations

$$
k U^{\prime}+m^{3} U^{\prime \prime \prime}+6 m U U^{\prime}=0
$$


Integrating of Eq.(4.3)with zero constant of integration, we have

$$
k U+m^{3} U^{\prime \prime}+3 m U^{2}=0 .
$$

Let us suppose that the solution of Eq.(4.4) can be expressed in the form Eq.(3.8). Substituting Eq.(3.8) into Eq.(4.4) and then by balancing the highest order derivative term and nonlinear term in result equation, the value of $N$ can be determined as 2. Therefore, Eq.(3.8) reduces to Eq.(3.10). Substituting Eq.(3.10) into Eq.(4.4), collecting all the terms with the same power of $\tan \left(\frac{\phi}{2}\right)$, we can obtain a set of algebraic equations for the unknowns $A_{0}, A_{1}, A_{2}, B_{1}, B_{2}, k, m$ :

$$
\begin{aligned}
& 6 A_{2}^{2} m+3 A_{2} b^{2} m^{3}-6 A_{2} b c m^{3}+3 A_{2} c^{2} m^{3}=0, \\
& 48 p A_{2}^{2} m+\ldots
\end{aligned}
$$

Solving the algebraic equations in the Mathematica, we obtain the following set of solutions:

Case 1: $A_{0}=-\frac{1}{2}(b-c) m^{2}\left(-b-c+2 a p+b p^{2}-c p^{2}\right), A_{1}=0, A_{2}=0, B_{1}=m^{2}\left(-a b-a c+2 a^{2} p-b^{2} p+c^{2} p+\right.$ $\left.3 a b p^{2}-3 a c p^{2}+b^{2} p^{3}-2 b c p^{3}+c^{2} p^{3}\right), B_{2}=-\frac{1}{2} m^{2}\left(-b-c+2 a p+b p^{2}-c p^{2}\right)^{2}, k=-\Delta m^{3}:$

For $b=c$ and $a=0$,

$$
U_{1}(\xi)=-2 m^{2} b^{2}\left[b \xi+c_{1}\right]^{-2}
$$

For $b=c$ and $a \neq 0$,

$$
U_{2}(\xi)=2 m^{2}\left(a^{2} p-a b\right)\left[p+c_{1} \exp (a \xi)-\frac{b}{a}\right]^{-1}-2 m^{2}(a p-b)^{2}\left[p+c_{1} \exp (a \xi)-\frac{b}{a}\right]^{-2} .
$$

For $\Delta>0$ and $b \neq c$,

$$
\begin{aligned}
& U_{3}(\xi)=-\frac{1}{2}(b-c) m^{2}\left(-b-c+2 a p+b p^{2}-c p^{2}\right) \\
+ & m^{2}\left(-a b-a c+2 a^{2} p-b^{2} p+c^{2} p+3 a b p^{2}-3 a c p^{2}+b^{2} p^{3}-2 b c p^{3}+c^{2} p^{3}\right) \\
\cdot & {\left[\frac{2}{b-c} \frac{c_{1} r_{1} \exp \left(r_{1} \xi\right)+c_{2} r_{2} \exp \left(r_{2} \xi\right)}{c_{1} \exp \left(r_{1} \xi\right)+c_{2} \exp \left(r_{2} \xi\right)}\right]^{-1} } \\
- & \frac{1}{2} m^{2}\left(-b-c+2 a p+b p^{2}-c p^{2}\right)^{2}\left[\frac{2}{b-c} \frac{c_{1} r_{1} \exp \left(r_{1} \xi\right)+c_{2} r_{2} \exp \left(r_{2} \xi\right)}{c_{1} \exp \left(r_{1} \xi\right)+c_{2} \exp \left(r_{2} \xi\right)}\right]^{-2}
\end{aligned}
$$

For $\Delta<0$ and $b \neq c$,

$$
\begin{aligned}
& U_{4}(\xi)=-\frac{1}{2}(b-c) m^{2}\left(-b-c+2 a p+b p^{2}-c p^{2}\right) \\
+ & m^{2}\left(-a b-a c+2 a^{2} p-b^{2} p+c^{2} p+3 a b p^{2}-3 a c p^{2}+b^{2} p^{3}-2 b c p^{3}+c^{2} p^{3}\right) \\
. & {\left[p+\frac{a}{b-c}+\frac{\sqrt{-\Delta}}{b-c} \frac{-c_{1} \sin \left(\frac{\sqrt{-\Delta}}{2} \xi\right)+c_{2} \cos \left(\frac{\sqrt{-\Delta}}{2} \xi\right)}{c_{1} \cos \left(\frac{\sqrt{-\Delta}}{2} \xi\right)+c_{2} \sin \left(\frac{\sqrt{-\Delta}}{2} \xi\right)}\right]^{-1} } \\
- & \frac{1}{2} m^{2}\left(-b-c+2 a p+b p^{2}-c p^{2}\right)^{2}\left[p+\frac{a}{b-c}+\frac{\sqrt{-\Delta}}{b-c} \frac{-c_{1} \sin \left(\frac{\sqrt{-\Delta}}{2} \xi\right)+c_{2} \cos \left(\frac{\sqrt{-\Delta}}{2} \xi\right)}{c_{1} \cos \left(\frac{\sqrt{-\Delta}}{2} \xi\right)+c_{2} \sin \left(\frac{\sqrt{-\Delta}}{2} \xi\right)}\right]^{-2} .
\end{aligned}
$$

Here $\xi=-\Delta m^{3} \frac{t^{\alpha}}{\alpha}+m \frac{x^{\beta}}{\beta}$.

Case 2: $A_{0}=-\frac{1}{6} m^{2}\left(2 a^{2}-b^{2}+c^{2}+6 a b p-6 a c p+3 b^{2} p^{2}-6 b c p^{2}+3 c^{2} p^{2}\right), A_{1}=0, A_{2}=0, B_{1}=m^{2}(-a b-a c+$ $\left.2 a^{2} p-b^{2} p+c^{2} p+3 a b p^{2}-3 a c p^{2}+b^{2} p^{3}-2 b c p^{3}+c^{2} p^{3}\right), B_{2}=-\frac{1}{2} m^{2}\left(-b-c+2 a p+b p^{2}-c p^{2}\right)^{2}, k=\Delta m^{3}:$

For $b=c$ and $a=0$,

$$
U_{5}(\xi)=-2 m^{2} b^{2}\left[b \xi+c_{1}\right]^{-2}
$$

For $b=c$ and $a \neq 0$,

$$
\begin{gathered}
U_{6}(\xi)=-\frac{1}{3} m^{2} a^{2}+2 m^{2}\left(a^{2} p-a b\right)\left[p+c_{1} \exp (a \xi)-\frac{b}{a}\right]^{-1} \\
-\quad 2 m^{2}(a p-b)^{2}\left[p+c_{1} \exp (a \xi)-\frac{b}{a}\right]^{-2} .
\end{gathered}
$$


For $\Delta>0$ and $b \neq c$,

$$
\begin{aligned}
& U_{7}(\xi)=-\frac{1}{6} m^{2}\left(2 a^{2}-b^{2}+c^{2}+6 a b p-6 a c p+3 b^{2} p^{2}-6 b c p^{2}+3 c^{2} p^{2}\right) \\
+ & m^{2}\left(-a b-a c+2 a^{2} p-b^{2} p+c^{2} p+3 a b p^{2}-3 a c p^{2}+b^{2} p^{3}-2 b c p^{3}+c^{2} p^{3}\right) \\
\cdot & {\left[\frac{2}{b-c} \frac{c_{1} r_{1} \exp \left(r_{1} \xi\right)+c_{2} r_{2} \exp \left(r_{2} \xi\right)}{c_{1} \exp \left(r_{1} \xi\right)+c_{2} \exp \left(r_{2} \xi\right)}\right]^{-1} } \\
- & \frac{1}{2} m^{2}\left(-b-c+2 a p+b p^{2}-c p^{2}\right)^{2}\left[\frac{2}{b-c} \frac{c_{1} r_{1} \exp \left(r_{1} \xi\right)+c_{2} r_{2} \exp \left(r_{2} \xi\right)}{c_{1} \exp \left(r_{1} \xi\right)+c_{2} \exp \left(r_{2} \xi\right)}\right]^{-2}
\end{aligned}
$$

For $\Delta<0$ and $b \neq c$,

$$
\begin{aligned}
& U_{8}(\xi)=-\frac{1}{6} m^{2}\left(2 a^{2}-b^{2}+c^{2}+6 a b p-6 a c p+3 b^{2} p^{2}-6 b c p^{2}+3 c^{2} p^{2}\right) \\
+ & m^{2}\left(-a b-a c+2 a^{2} p-b^{2} p+c^{2} p+3 a b p^{2}-3 a c p^{2}+b^{2} p^{3}-2 b c p^{3}+c^{2} p^{3}\right) \\
\cdot & {\left[p+\frac{a}{b-c}+\frac{\sqrt{-\Delta}}{b-c} \frac{c_{1} \sin \left(\frac{\sqrt{-\Delta}}{2} \xi\right)+c_{2} \cos \left(\frac{\sqrt{-\Delta}}{2} \xi\right)}{c_{1} \cos \left(\frac{\sqrt{-\Delta}}{2} \xi\right)+c_{2} \sin \left(\frac{\sqrt{-\Delta}}{2} \xi\right)}\right]^{-1} } \\
- & \frac{1}{2} m^{2}\left(-b-c+2 a p+b p^{2}-c p^{2}\right)^{2}\left[p+\frac{a}{b-c}\right. \\
+ & \left.\frac{\sqrt{-\Delta}}{b-c} \frac{-c_{1} \sin \left(\frac{\sqrt{-\Delta}}{2} \xi\right)+c_{2} \cos \left(\frac{\sqrt{-\Delta}}{2} \xi\right)}{c_{1} \cos \left(\frac{\sqrt{-\Delta}}{2} \xi\right)+c_{2} \sin \left(\frac{\sqrt{-\Delta}}{2} \xi\right)}\right]^{-2} .
\end{aligned}
$$

Here $\xi=\Delta m^{3} \frac{t^{\alpha}}{\alpha}+m \frac{x^{\beta}}{\beta}$.

Case 3: $A_{0}=-\frac{1}{2}(b-c) m^{2}\left(-b-c+2 a p+b p^{2}-c p^{2}\right), A_{1}=(b-c) m^{2}(a+b p-c p), A_{2}=-\frac{1}{2}(b-c)^{2} m^{2}, B_{1}=$ $0, B_{2}=0, k=-\Delta m^{3}$ :

For $\Delta>0$ and $b \neq c$,

$$
\begin{array}{r}
U_{9}(\xi)=-\frac{1}{2}(b-c) m^{2}\left(-b-c+2 a p+b p^{2}-c p^{2}\right) \\
+\quad 2 m^{2}(a+b p-c p)\left[\frac{c_{1} r_{1} \exp \left(r_{1} \xi\right)+c_{2} r_{2} \exp \left(r_{2} \xi\right)}{c_{1} \exp \left(r_{1} \xi\right)+c_{2} \exp \left(r_{2} \xi\right)}\right] \\
-\quad 2 m^{2}\left[\frac{c_{1} r_{1} \exp \left(r_{1} \xi\right)+c_{2} r_{2} \exp \left(r_{2} \xi\right)}{c_{1} \exp \left(r_{1} \xi\right)+c_{2} \exp \left(r_{2} \xi\right)}\right]^{2}
\end{array}
$$

For $\Delta<0$ and $b \neq c$,

$$
\begin{aligned}
& U_{10}(\xi)=-\frac{1}{2}(b-c) m^{2}\left(-b-c+2 a p+b p^{2}-c p^{2}\right) \\
& +\quad(b-c) m^{2}(a+b p-c p)\left[p+\frac{a}{b-c}+\frac{\sqrt{-\Delta}}{b-c} \frac{c_{1} \sin \left(\frac{\sqrt{-\Delta}}{2} \xi\right)+c_{2} \cos \left(\frac{\sqrt{-\Delta}}{2} \xi\right)}{c_{1} \cos \left(\frac{\sqrt{-\Delta}}{2} \xi\right)+c_{2} \sin \left(\frac{\sqrt{-\Delta}}{2} \xi\right)}\right] \\
& +\quad-\frac{1}{2}(b-c)^{2} m^{2}\left[p+\frac{a}{b-c}+\frac{\sqrt{-\Delta}}{b-c} \frac{-c_{1} \sin \left(\frac{\sqrt{-\Delta}}{2} \xi\right)+c_{2} \cos \left(\frac{\sqrt{-\Delta}}{2} \xi\right)}{c_{1} \cos \left(\frac{\sqrt{-\Delta}}{2} \xi\right)+c_{2} \sin \left(\frac{\sqrt{-\Delta}}{2} \xi\right)}\right]^{2}
\end{aligned}
$$

Here $\xi=-\Delta m^{3} \frac{t^{\alpha}}{\alpha}+m \frac{x^{\beta}}{\beta}$.

Case 4: $A_{0}=-\frac{1}{6} m^{2}\left(2 a^{2}-b^{2}+c^{2}+6 a b p-6 a c p+3 b^{2} p^{2}-6 b c p^{2}+3 c^{2} p^{2}\right), A_{1}=(b-c) m^{2}(a+b p-c p), A_{2}=$ $-\frac{1}{2}(b-c)^{2} m^{2}, B_{1}=0, B_{2}=0, k=\Delta m^{3}$ :

For $b=c$ and $a \neq 0$,

$$
U_{11}(\xi)=-\frac{1}{3} m^{2} a^{2}
$$


For $\Delta>0$ and $b \neq c$,

$$
\begin{aligned}
& U_{12}(\xi)=-\frac{1}{6} m^{2}\left(2 a^{2}-b^{2}+c^{2}+6 a b p-6 a c p+3 b^{2} p^{2}-6 b c p^{2}+3 c^{2} p^{2}\right) \\
+ & 2 m^{2}(a+b p-c p)\left[\frac{c_{1} r_{1} \exp \left(r_{1} \xi\right)+c_{2} r_{2} \exp \left(r_{2} \xi\right)}{c_{1} \exp \left(r_{1} \xi\right)+c_{2} \exp \left(r_{2} \xi\right)}\right] \\
- & 2 m^{2}\left[\frac{c_{1} r_{1} \exp \left(r_{1} \xi\right)+c_{2} r_{2} \exp \left(r_{2} \xi\right)}{c_{1} \exp \left(r_{1} \xi\right)+c_{2} \exp \left(r_{2} \xi\right)}\right]^{2}
\end{aligned}
$$

For $\Delta<0$ and $b \neq c$,

$$
\begin{gathered}
U_{13}(\xi)=-\frac{1}{6} m^{2}\left(2 a^{2}-b^{2}+c^{2}+6 a b p-6 a c p+3 b^{2} p^{2}-6 b c p^{2}+3 c^{2} p^{2}\right) \\
+m^{2}(a+b p-c p)\left[p(b-c)+a+\sqrt{-\Delta} \frac{-c_{1} \sin \left(\frac{\sqrt{-\Delta}}{2} \xi\right)+c_{2} \cos \left(\frac{\sqrt{-\Delta}}{2} \xi\right)}{c_{1} \cos \left(\frac{\sqrt{-\Delta}}{2} \xi\right)+c_{2} \sin \left(\frac{\sqrt{-\Delta}}{2} \xi\right)}\right] \\
-\frac{1}{2} m^{2}\left[p(b-c)+a+\sqrt{-\Delta} \frac{-c_{1} \sin \left(\frac{\sqrt{-\Delta}}{2} \xi\right)+c_{2} \cos \left(\frac{\sqrt{-\Delta}}{2} \xi\right)}{c_{1} \cos \left(\frac{\sqrt{-\Delta}}{2} \xi\right)+c_{2} \sin \left(\frac{\sqrt{-\Delta}}{2} \xi\right)}\right]^{2} .
\end{gathered}
$$

Here $\xi=\Delta m^{3} \frac{t^{\alpha}}{\alpha}+m \frac{x^{\beta}}{\beta}$.

The solution $u_{13}(x, t)$ of the Eq.(4.1) is simulated in Fig.7-Fig.8 as periodic waves solutions. 3D plot of the obtained solution $u_{13}(x, t)$ is given for parameters $\alpha=0.5, \beta=1, m=0.5, a=0.5, b=0.25, c=1, c_{1}=1, c_{2}=3$ and $p=1$ in Fig.7. Fig.8 demonstrate the same solution with 2D plot for $-40 \leq x \leq 40$ at $t=1$.

\section{Analytic solutions to the conformable space-time fractional Burger's equation}

Conformable space-time fractional Burger's equation is given in the following form[31]

$$
T_{t}^{\alpha} u+u T_{x}^{\beta} u-T_{x}^{\beta} T_{x}^{\beta} u=0,0<\alpha \leq 1,0<\beta \leq 1 .
$$

Let us consider the following transformation

$$
u(x, t)=U(\xi), \xi=k \frac{t^{\alpha}}{\alpha}+m \frac{x^{\beta}}{\beta},
$$

where $k, m$ are constants. Substituting Eq.(5.2) into Eq.(5.1) we obtain the following differential equations

$$
k U^{\prime}+m U U^{\prime}-m^{2} U^{\prime \prime}=0 .
$$

Integrating of Eq.(5.3)with zero constant of integration, we have

$$
k U+\frac{m}{2} U^{2}-m^{2} U^{\prime}=0 .
$$

Let us suppose that the solution of Eq.(5.4) can be expressed in the form Eq.(3.8). Substituting Eq.(3.8) into Eq.(5.4) and then by balancing the highest order derivative term and nonlinear term in result equation, the value of $N$ can be determined as 1. Therefore, Eq.(3.8) reduces to

$$
U(\xi)=A_{0}+A_{1}\left[p+\tan \left(\frac{\phi(\xi)}{2}\right)\right]+B_{1}\left[p+\tan \left(\frac{\phi(\xi)}{2}\right)\right]^{-1} .
$$

Substituting Eq.(5.5) into Eq.(5.4), collecting all the terms with the same power of $\tan \left(\frac{\phi}{2}\right)$, we can obtain a set of algebraic equations for the unknowns $A_{0}, A_{1}, B_{1}, k, m$ :

$$
\begin{aligned}
& A_{1}^{2} m+A_{1} b m^{2}-A_{1} c m^{2}=0, \\
& 2 A_{1} k+2 A_{0} A_{1} m-2 a A_{1} m^{2}+4 A_{1}^{2} m p+2 A_{1} b m^{2} p-2 A_{1} c m^{2} p=0, \\
& 2 A_{0} k+A_{0}^{2} m+6 A_{1}^{2} m p^{2}+2 A_{1} B_{1} m+6 A_{1} k p-A_{1} b m^{2}-A_{1} c m^{2}-b B_{1} m^{2} \\
+ & B_{1} c m^{2}-4 a A_{1} m^{2} p+A_{1} b m^{2} p^{2}-A_{1} c m^{2} p^{2}+6 A_{0} A_{1} m p=0, \\
& 2 B_{1} k+4 A_{1}^{2} m p^{3}+2 A_{0} B_{1} m+4 A_{0} k p+2 a B_{1} m^{2}+6 A_{1} k p^{2}+2 A_{0}^{2} m p \\
+ & 6 A_{0} A_{1} m p^{2}-2 A_{1} b m^{2} p-2 A_{1} c m^{2} p-2 a A_{1} m^{2} p^{2}+4 A_{1} B_{1} m p=0, \\
& B_{1}^{2} m+A_{0}^{2} m p^{2}+A_{1}^{2} m p^{4}+2 B_{1} k p+b B_{1} m^{2}+B_{1} c m^{2}+2 A_{0} k p^{2}+2 A_{1} k p^{3} \\
+ & 2 A_{0} A_{1} m p^{3}+2 A_{1} B_{1} m p^{2}-A_{1} b m^{2} p^{2}-A_{1} c m^{2} p^{2}+2 A_{0} B_{1} m p=0 .
\end{aligned}
$$


Solving the algebraic equations in the Mathematica, we obtain the following set of solutions:

Case 1: $A_{0}=-a m \pm \sqrt{m^{2} \Delta}-m p(b-c), A_{1}=0, B_{1}=m\left(-b-c+2 a p+b p^{2}-c p^{2}\right), k=\mp m \sqrt{m^{2} \Delta}$ :

For $b=c$ and $a=0$,

$$
U_{1}(\xi)=-2 b m\left[b \xi+c_{1}\right]^{-1}
$$

For $b=c$ and $a \neq 0$,

$$
U_{2,3}(\xi)=-a m \pm \sqrt{m^{2} a^{2}}+2 m(a p-b)\left[p+c_{1} \exp (a \xi)-\frac{b}{a}\right]^{-1}
$$

For $\Delta>0$ and $b \neq c$,

$$
\begin{gathered}
U_{4,5}(\xi)=-a m \pm \sqrt{m^{2} \Delta}-m p(b-c) \\
+\quad m\left(-b-c+2 a p+b p^{2}-c p^{2}\right)\left[\frac{2}{b-c} \frac{c_{1} r_{1} \exp \left(r_{1} \xi\right)+c_{2} r_{2} \exp \left(r_{2} \xi\right)}{c_{1} \exp \left(r_{1} \xi\right)+c_{2} \exp \left(r_{2} \xi\right)}\right]^{-1}
\end{gathered}
$$

For $\Delta<0$ and $b \neq c$,

$$
\begin{aligned}
& U_{6,7}(\xi)=-a m \pm \sqrt{m^{2} \Delta}-m p(b-c) \\
& +\quad m\left(-b-c+2 a p+b p^{2}-c p^{2}\right)\left[p+\frac{a}{b-c}+\frac{\sqrt{-\Delta}}{b-c} \frac{-c_{1} \sin \left(\frac{\sqrt{-\Delta}}{2} \xi\right)+c_{2} \cos \left(\frac{\sqrt{-\Delta}}{2} \xi\right)}{c_{1} \cos \left(\frac{\sqrt{-\Delta}}{2} \xi\right)+c_{2} \sin \left(\frac{\sqrt{-\Delta}}{2} \xi\right)}\right]^{-1} .
\end{aligned}
$$

Here $\xi=\mp m \sqrt{m^{2} \Delta} \frac{t^{\alpha}}{\alpha}+m \frac{x^{\beta}}{\beta}$.

Case 2: $A_{0}=a m \pm \sqrt{m^{2} \Delta}+m p(b-c), A_{1}=-m(b-c), B_{1}=0, k=\mp m \sqrt{m^{2} \Delta}:$

For $\Delta>0$ and $b \neq c$,

$$
\begin{gathered}
U_{8,9}(\xi)=a m \pm \sqrt{m^{2} \Delta}+m p(b-c) \\
-\quad 2 m\left[\frac{c_{1} r_{1} \exp \left(r_{1} \xi\right)+c_{2} r_{2} \exp \left(r_{2} \xi\right)}{c_{1} \exp \left(r_{1} \xi\right)+c_{2} \exp \left(r_{2} \xi\right)}\right] .
\end{gathered}
$$

For $\Delta<0$ and $b \neq c$,

$$
\begin{gathered}
U_{10,11}(\xi)=a m \pm \sqrt{m^{2} \Delta}+m p(b-c) \\
-m\left[p(b-c)+a+\sqrt{-\Delta} \frac{-c_{1} \sin \left(\frac{\sqrt{-\Delta}}{2} \xi\right)+c_{2} \cos \left(\frac{\sqrt{-\Delta}}{2} \xi\right)}{c_{1} \cos \left(\frac{\sqrt{-\Delta}}{2} \xi\right)+c_{2} \sin \left(\frac{\sqrt{-\Delta}}{2} \xi\right)}\right] .
\end{gathered}
$$

Here $\xi=\mp m \sqrt{m^{2} \Delta} \frac{t^{\alpha}}{\alpha}+m \frac{x^{\beta}}{\beta}$.

The solution $u_{5}(x, t)$ in Eq.(5.8) is simulated in Fig.9-Fig.10. These figures show kink wave solutions. Figs.9 and 10 are $3 \mathrm{D}$ and $2 \mathrm{D}$ plots of the traveling wave solution $u_{5}(x, t)$ and $u_{5}(x, 1)$ in Eq.(5.8) for $\alpha=0.75, \beta=1, \theta=0.5$, $m=0.5, a=2, b=5, c=2, c_{1}=1, c_{2}=1$ and $p=0.2$. 


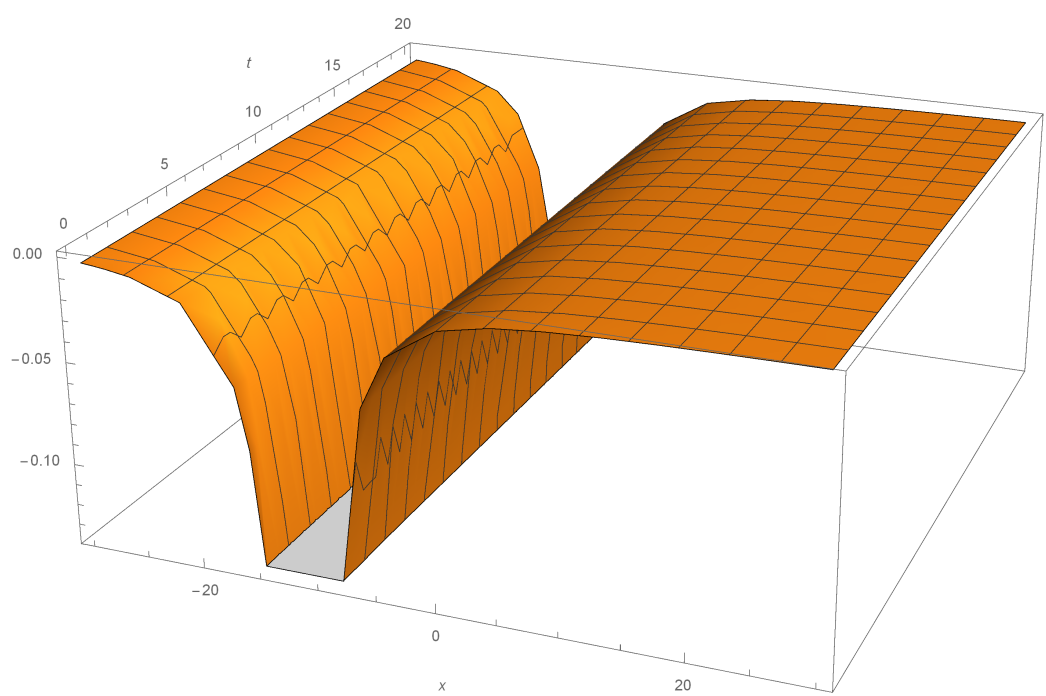

Figure 1. 3D plot of the obtained traveling wave solution $u_{2}(x, 1, t)$ in Eq.(3.12).

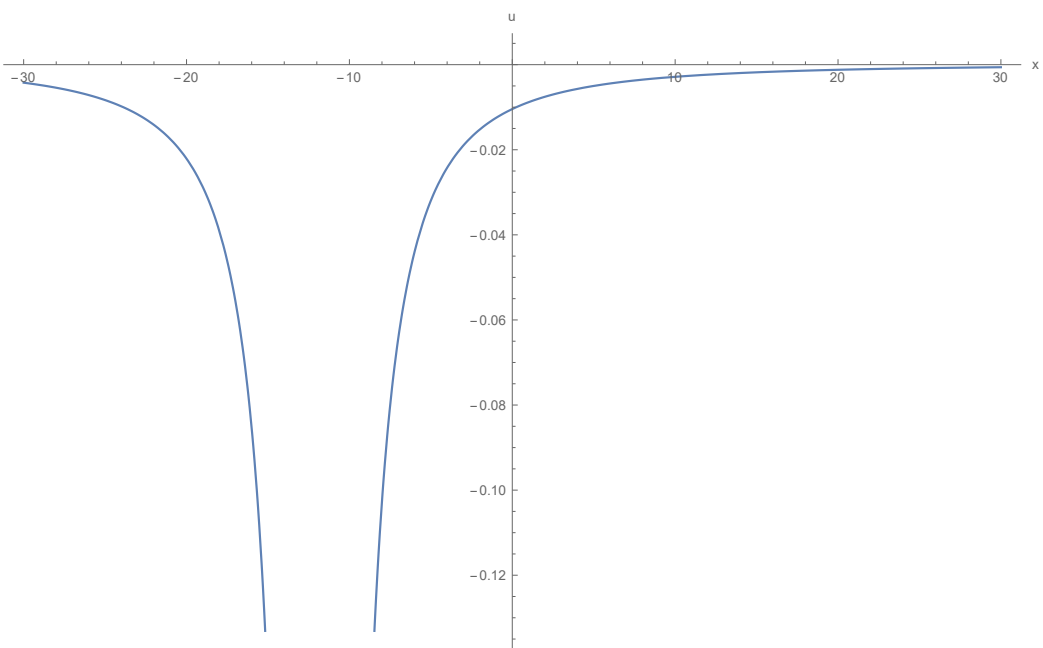

Figure 2. 2D plot of the obtained traveling wave solution $u_{2}(x, 1,1)$ in Eq.(3.12).

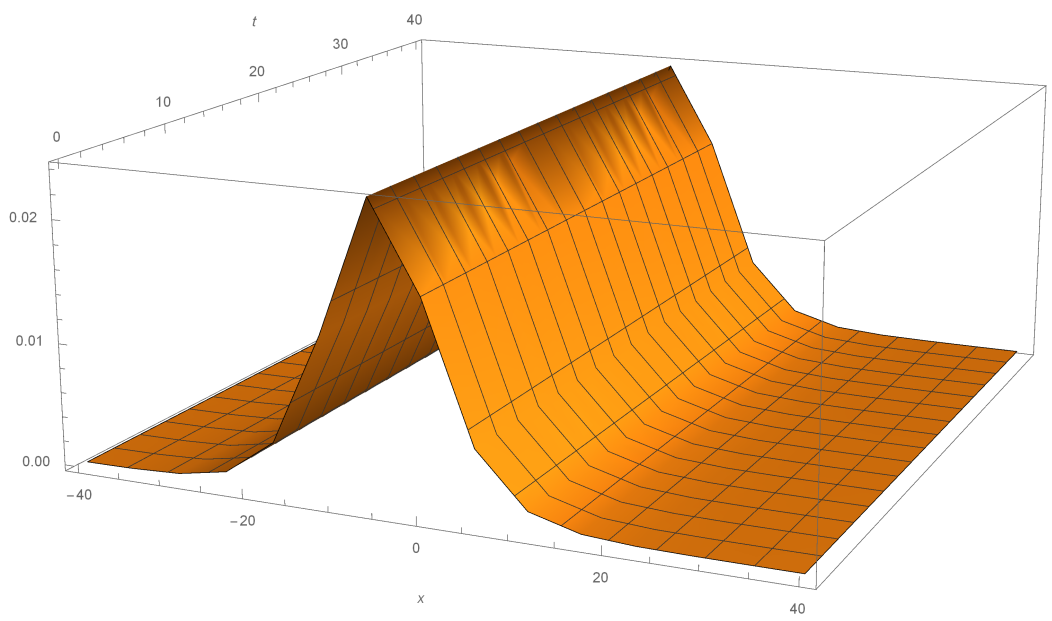

Figure 3. 3D plot of the obtained traveling wave solution $u_{3}(x, 1, t)$ in Eq.(3.13). 


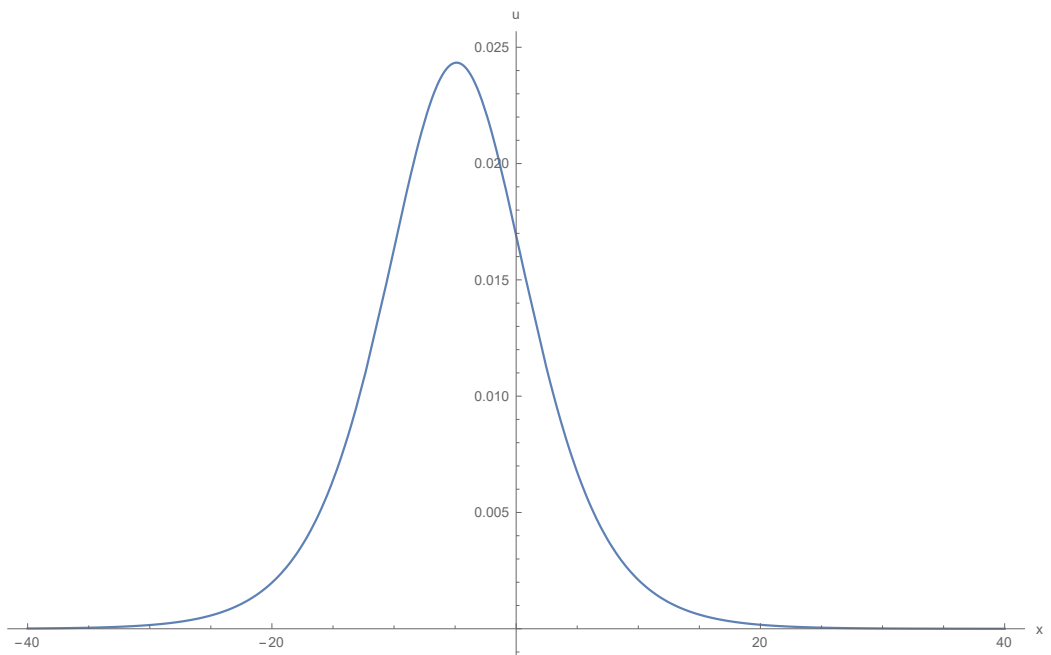

Figure 4. 2D plot of the obtained traveling wave solution $u_{3}(x, 1,1)$ in Eq.(3.13).

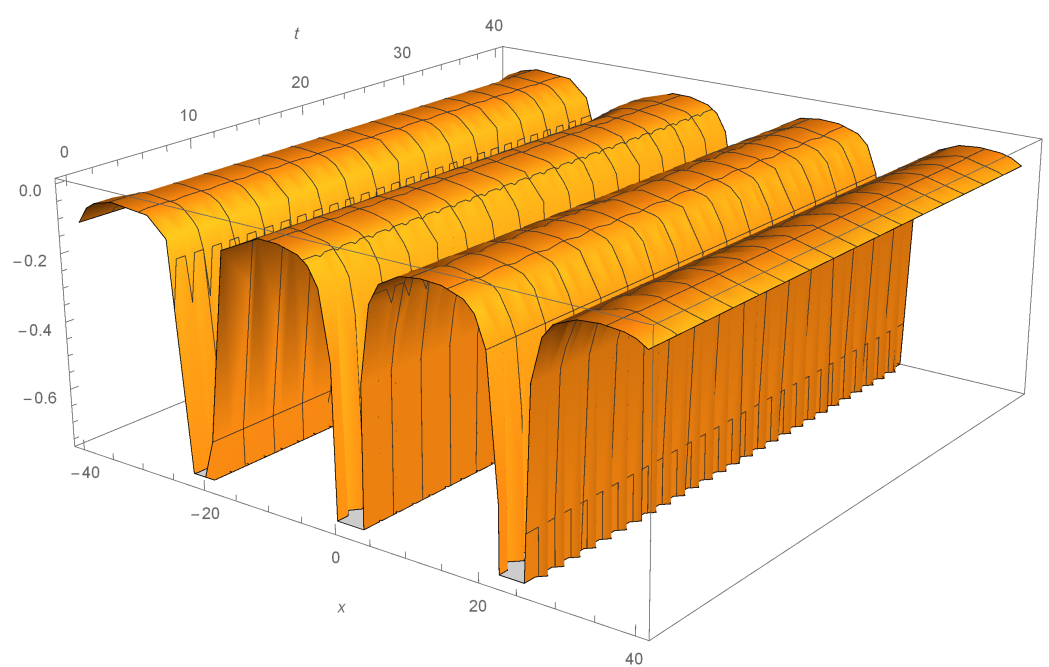

Figure 5. 3D plot of the obtained traveling wave solution $u_{4}(x, 1, t)$ in Eq.(3.14).

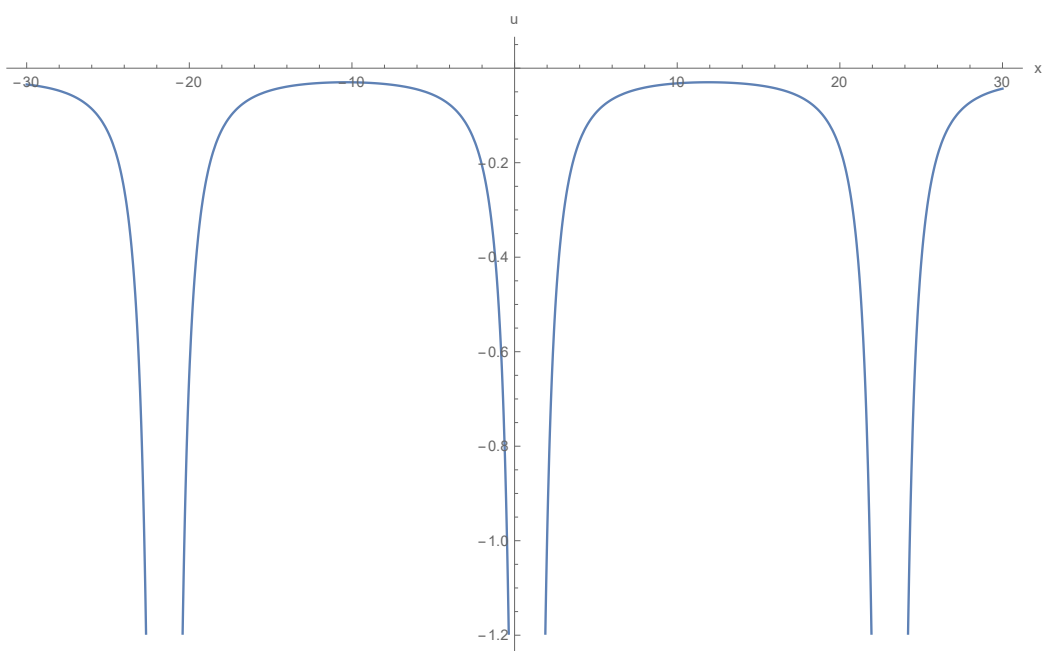

Figure 6. 2D plot of the obtained traveling wave solution $u_{4}(x, 1,1)$ in Eq.(3.14). 


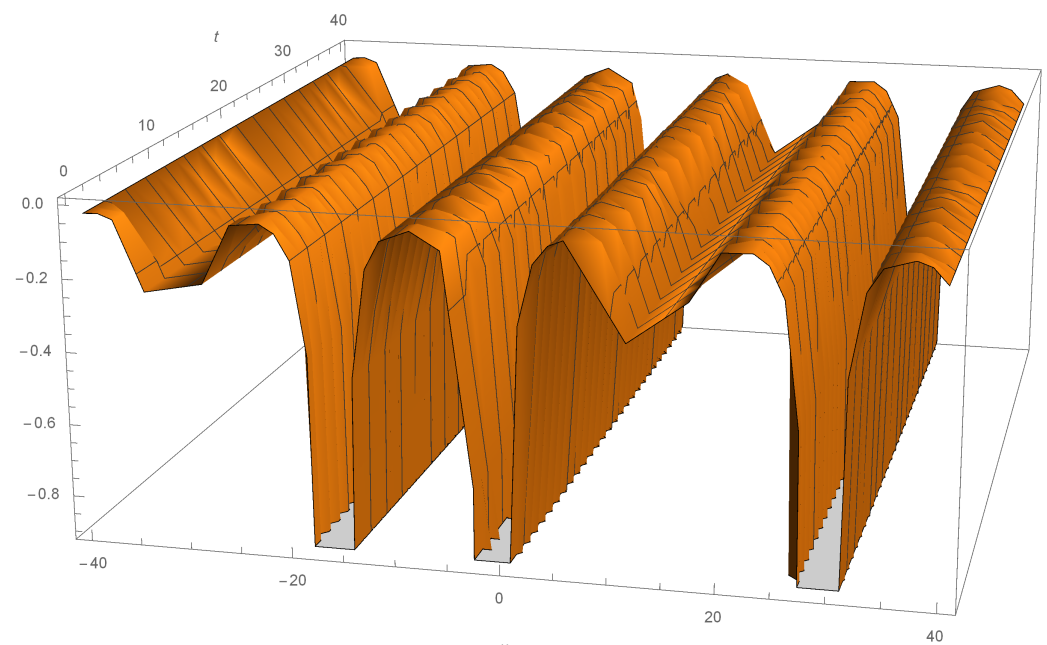

Figure 7. 3D plot of the obtained traveling wave solution $u_{13}(x, t)$ in Eq.(4.17).

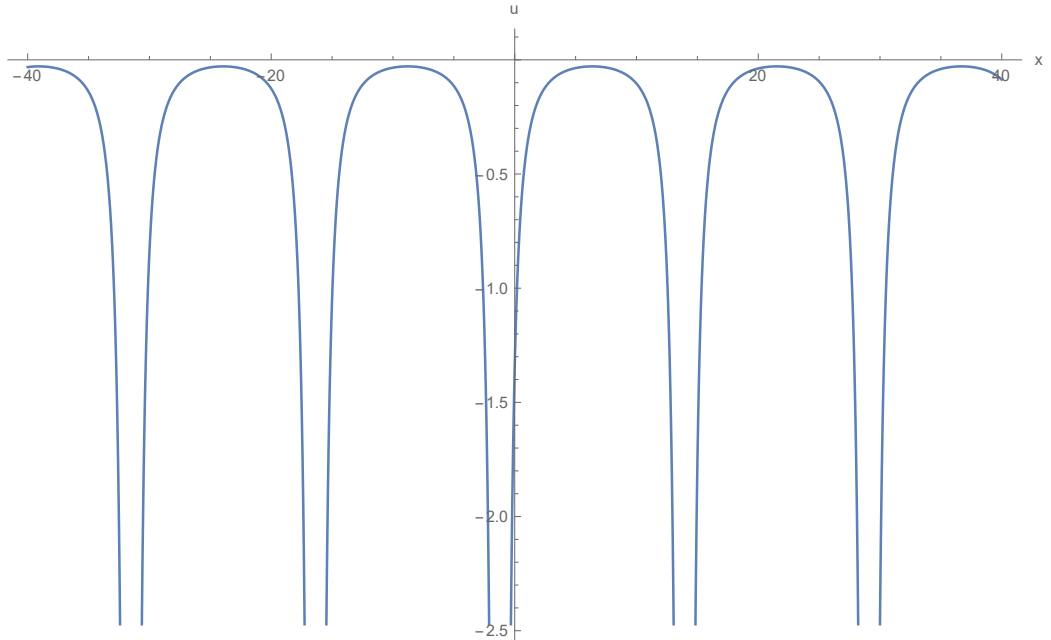

Figure 8. 2D plot of the obtained traveling wave solution $u_{13}(x, 1)$ in Eq.(4.17).

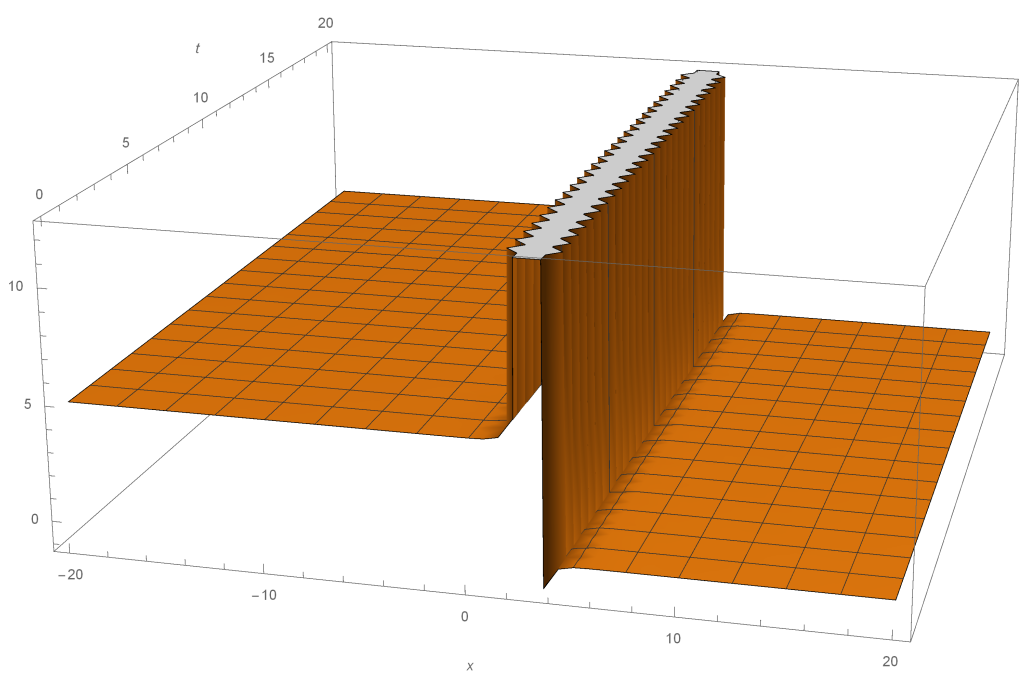

Figure 9. 3D plot of the obtained traveling wave solution $u_{5}(x, t)$ in Eq.(5.8). 


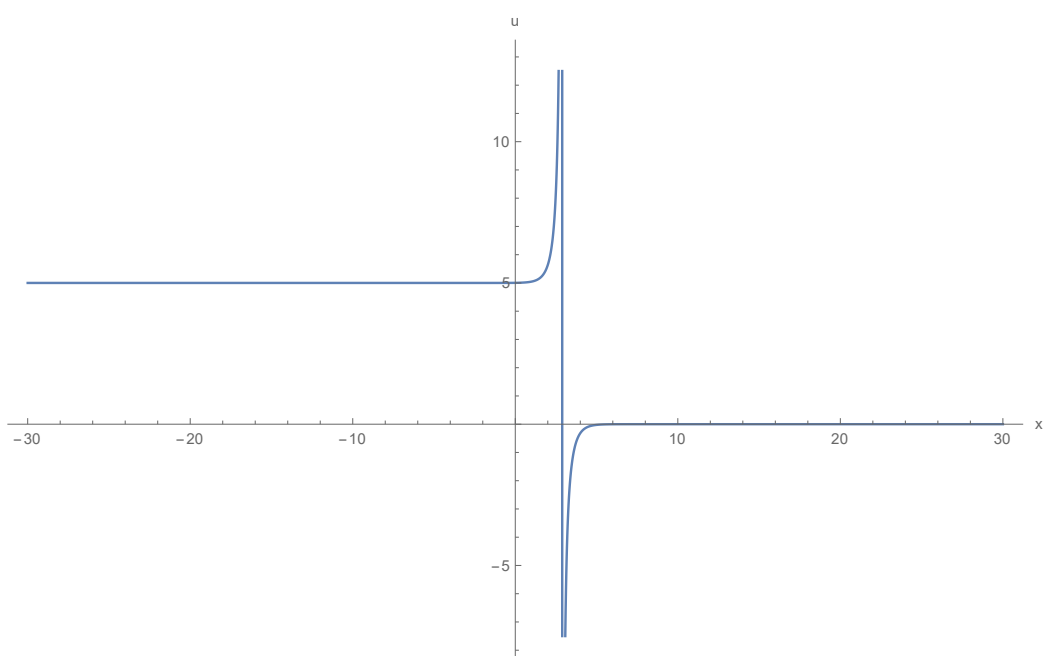

Figure 10. 2D plot of the obtained traveling wave solution $u_{5}(x, 1)$ in Eq.(5.8).

\section{Conclusion}

The fundamental goal of the paper has been to construct an approximation to the solution of the conformable space-time fractional (2+1)-dimensional breaking soliton, third-order KdV and Burger's equations by SITEM. The obtained solutions are traveling wave solutions of the conformable space-time fractional (2+1)-dimensional breaking soliton, third-order KdV and Burger's equations. These equations have been converted into its equivalent nonlinear ordinary differential equation by using fractional complex transformation. Solutions of the obtained nonlinear ordinary differential equation have been seek in the form of the summation of the function $p+\tan \left(\frac{\phi(\xi)}{2}\right)$. Substituting the summation of the function $p+\tan \left(\frac{\phi(\xi)}{2}\right)$ into the nonlinear ordinary differential equation and equalizing coefficients of the term with the same degree, nonlinear algebraic system is obtained. Solving the nonlinear algebraic system, we have the traveling wave solutions.

There are many types of traveling waves that are of particular interest in solitary wave theory. Three of these types are the solitary waves, the periodic waves and the kink waves. The solitary waves are asymptotically zero at large distances, the periodic waves have periodicity, the kink waves rise or descend from one asymptotic state to another. The 3D and 2D graphics of the obtained solutions have been presented in the paper. Figs.1- 2, Figs.9-10 show kink waves solutions, Figs.5- 6, Figs.7-8 have periodic waves solutions and Figs.3-4 give solitary waves solutions. This method changes the given difficult problems into simple one and solve easily by using MATLAB programming. The obtained solutions are new and have not been reported in former literature. The method can also be applied to other nonlinear fractional partial differential equations.

\section{Acknowledgements}

The authors would like to express their sincere thanks to the editor and the anonymous reviewers for their helpful comments and suggestions.

\section{Funding}

There is no funding for this work.

\section{Availability of data and materials}

Not applicable.

\section{Competing interests}

The authors declare that they have no competing interests. 


\section{Author's contributions}

All authors contributed equally to the writing of this paper. All authors read and approved the final manuscript.

\section{References}

[1] Bagley, R.L., Torvik, P.J.: On the appearance of the fractional derivative in the behavior of real materials. J. Appl. Mech. 51, 294-298 (1984). https:/ / doi.org/10.1115/1.3167615

[2] Podlubny, I.: Geometric and physical interpretation of fractional integration and fractional differentiation. Fract. Calculus. App. Anal. 5, 367 - 386 (2002).

[3] Mainardi, F.: Fractional calculus: Some basic problems in continuum and statistical mechanics, In: A. Carpinteri, F. Mainardi, Editors, Fractals and Fractional Calculus in Continuum Mechanics. Springer-Verlag, New York (1997).

[4] Thomas, M.D., Bamforth,P.B.: Modelling chloride diffusion in concrete: Effect of fly ash and slag. Cem. Concr. Res. 29 (4), 487-495 (1999).

[5] Khitab, A., Lorente, S., Ollivier, J.P.: Predictive model for chloride penetration through concrete. Mag. Concr. Res. 57 (9), 511-520 (2005). https:/ / doi.org/10.1680/macr.2005.57.9.511

[6] Sahoo, S., Saha Ray S.: New travelling wave and anti-kink wave solutions of space-time fractional (3+1)-Dimensional Jimbo-Miwa equation. Chin. J. Phys. 67, 79-85 (2020). https:/ / doi.org/10.1016/j.cjph.2020.04.016.

[7] Kim, H., Sakthivel, R., Debbouchecd, A., Torres, Delfim F.M.: Traveling wave solutions of some important Wick-type fractional stochastic nonlinear partial differential equation. Chaos soliton fract. 131, 109542, (2020). https://doi.org/10.1016/j.chaos.2019.109542.

[8] Zulfiqar, A., Ahmad, J.: Exact solitary wave solutions of fractional modified Camassa-Holm equation using an efficient method. Alexandria Eng. J. 59 (5), 3565-3574 (2020). https:/ / doi.org/10.1016/j.aej.2020.06.002.

[9] Sulaimana, T. A., Bulut, H.: Boussinesq equations: M-fractional solitary wave solutions and convergence analysis. JOES 4 (1), 1-6 (2019). https:/ / doi.org/10.1016/j.joes.2018.12.001.

[10] Dianchen, L. Y., Arshad, L. M., Xu, X.: New Exact Traveling Wave Solutions of the Unstable Nonlinear Schrödinger Equations and their Applications. Optik 165386 (2020). https:/ / doi.org/10.1016/j.ijleo.2020.165386.

[11] Guo, S., Mei, L., Zhou, Y.: The compound $\left(G^{\prime} / G\right)$-expansion method and double non-traveling wave solutions of $(2+1)$-dimensional nonlinear partial differential equations. Comput. Math. Appl. 69, 804-816 (2015). https://doi.org/10.1016/j.camwa.2015.02.016.

[12] Bekir,A., Uygun, F.: Exact travelling wave solutions of nonlinear evolution equations by using $\left(G^{\prime} / G\right)$-expansion method. Arab J. Math. Sci. 18, 73-85 (2012). https://doi.org/10.1016/j.ajmsc.2011.08.002.

[13] Dai, C., Zhang, J.: Chaotic behaviors in the $(2+1)$-dimensional breaking soliton system. Chaos soliton fract. 39, 889-894 (2009). https://doi.org/10.1016/j.chaos.2007.01.063.

[14] Ping, Z.: New exact solutions to breaking soliton equations and Whitham-Broer-Kaup equations. Appl. Math. Comput. 217, 1688-1696 (2010). https:/ / doi.org/10.1016/j.amc.2009.09.062.

[15] Tascan, F., Bekir, A.: Analytic solutions of the $(2+1)$-dimensional nonlinear evolution equations using the sine-cosine method. Appl. Math. Comput. 215, 3134-3139 (2009). https://doi.org/10.1016/j.amc.2009.09.027.

[16] Zhang, S.: A further improved extended Fan sub-equation method for (2+1)-dimensional breaking soliton equations. Appl. Math. Comput. 199, 259-267 (2008). https:/ / doi.org/10.1016/j.amc.2007.09.052.

[17] Xia,T., Xiong, S.: Exact solutions of (2+1)-dimensional Bogoyavlenskii's breaking soliton equation with symbolic computation. Comput. Math. Appl. 60, 919-923 (2010). https://doi.org/10.1016/j.camwa.2010.05.037.

[18] Zhao, Z., Dai, Z., Mu, G.: The breather-type and periodic-type soliton solutions for the (2+1)-dimensional breaking soliton equation. Comput. Math. Appl. 61, 2048-2052 (2011). https://doi.org/10.1016/j.camwa.2010.08.065. 
[19] Zheng, B.: A new fractional Jacobi elliptic equation method for solving fractional partial differential equations. Adv. Differ. Equ. 228, 1-11 (2014). https:/ /doi.org/10.1186/1687-1847-2014-228.

[20] Choi, J. H., Kim, H.: Soliton solutions for the space-time nonlinear partial differential equations with fractional-orders. Chinese J. Phys. 55, 556-565 (2017). https:/ / doi.org/10.1016/j.cjph.2016.10.019.

[21] Kaplan, M., Akbulut, A., Bekir, A.: Solving Space-Time Fractional Differential Equations by Using Modified Simple Equation Method. Commun. Theor. Phys. 65, 563-568 (2016). https://doi.org/10.1088/0253-6102/65/5/563.

[22] Li,C., Zhao, M.: Analytical solutions of the (2+1)-dimensional space-time fractional Bogoyavlenskii's breaking soliton equation. Appl. Math. Lett. 84, 13-18 (2018). https://doi.org/10.1016/j.aml.2018.04.011.

[23] Mohyud-Din, S. T., Bibi, S.: Exact solutions for nonlinear fractional differential equations using exponential rational function method. Opt. Quant. Electron. 49, 1-12 (2017). https:/ / doi.org/10.1007/s11082-017-0895-9

[24] Shang, N., Zheng, B.: Exact Solutions for Three Fractional Partial Differential Equations by the $\left(G^{\prime} / G\right)$ Method. IJAM 43, 1-6 (2013).

[25] Salas, A. H., Gomez, A.: Exact Solutions for a Third-Order KdV Equation with Variable Coefficients and Forcing Term. Math. Probl. Eng. 2009, 1-13 (2009). https:/ / doi.org/10.1155/2009/737928.

[26] Wazzan, L.: Exact solutions for the family of third order Korteweg de-Vries equations. Commun. Appl. Anal. 2016, 108-117 (2016). https:/ / doi.org/10.5899/2016/cna-00242.

[27] Seadawy, A. R., Sayed, A.: Traveling Wave Solutions of the Benjamin-Bona-Mahony Water Wave Equations. Abstr. Appl. Anal. 2014, 1-7 (2014). https:/ / doi.org/10.1155/2014/926838.

[28] Raslan, K. R., EL-Danaf, T. S., Ali, K. K.: New numerical treatment for solving the KDV equation. JACM 1, 1-12 (2017).

[29] Demiray, S. T., Pandir, Y., Bulut, H.: Generalized Kudryashov Method for Time-Fractional Differential Equations. Abstr. Appl. Anal. 2014 1-13 (2014). https:/ / doi.org/10.1155/2014/901540.

[30] Cole, J.D.: On a quasi-linear parabolic equation occurring in aerodynamics. Quart. Appl. Math. 9, 225-236 (1951). https://doi.org/10.1090/qam/42889.

[31] Xiea, S., Heo, S., Kimc, S., Woo, G., Yi, S.:Numerical solution of one-dimensional Burgers' equation using reproducing kernel function. J. Comput. Appl. Math. 214, 417-434 (2008). https://doi.org/10.1016/j.cam.2007.03.010.

[32] AL-Jawary, M. A., Azeez, M. M., Radhi, G. H.: Analytical and numerical solutions for the nonlinear Burgers and advection-diffusion equations by using a semi-analytical iterative method. Comput. Math. Appl. 76, 155-171 (2018). https://doi.org/10.1016/j.camwa.2018.04.010.

[33] Huda, M. A., Akbar, M. A., Shanta, S. S.: The new types of wave solutions of the Burger's equation and the BenjaminBona-Mahony equation. JOES 3, 1-10 (2018). https:/ / doi.org/10.1016/j.joes.2017.11.002.

[34] Liu, H., Zhang, T.: A note on the improved $\tan (\phi(\xi) / 2)$-expansion method. Optik 131, $273-278$ (2017). https://doi.org/10.1016/j.ijleo.2016.11.029.

[35] Khalil, R., Horani, M. A., Yousef, A., Sababheh, M.: A new defnition of fractional derivative. J. Comput. Appl. Math. 264 , 65-70 (2014). https://doi.org/10.1016/j.cam.2014.01.002.

[36] Bogoyavlenskii, O.I.: Breaking solitons in (2 + 1)-dimensional integrable equations. Russian Math. Surveys 45, 1-86 (1990). https://doi.org/10.1070/RM1990v045n04ABEH002377.

\section{Affiliations}

H. Cerdik Yaslan

AdDRESS: Pamukkale University, Department of Mathematics, 20070 Denizli, Turkey.

E-MAIL: hcerdik@pau.edu.tr

ORCID ID: 0000-0002-3243-3703 